\title{
Article
}

\section{A new species of Perenniporia (Polyporales, Basidiomycota) from Thailand}

\author{
Ji XH' ${ }^{1}$, Thawthong $A^{2}$ and $W u F^{1}$ \\ ${ }^{1}$ Institute of Microbiology, PO Box 61, Beijing Forestry University, Beijing 100083, China \\ ${ }^{2}$ Center of Excellence in Fungal Research, Mae Fah Luang University, Chiang Rai 57100, Thailand
}

Ji XH, Thawthong A, Wu F 2017 - A new species of Perenniporia (Polyporales, Basidiomycota) from Thailand. Mycosphere 8(8), 1102-1107, Doi 10.5943/mycosphere/8/8/10

\begin{abstract}
Perenniporia chiangraiensis is introduced as a new species from Thailand based on morphological characters and molecular data, and it is characterized by perennial and pileate basidiocarps with concentric sulcate pileal surface and a white pore surface, distinctly stratified tubes, a dimitic hyphal system with strongly dextrinoid skeletal hyphae, the presence of dendrohyphidia, and ellipsoid, truncate, thick-walled, non-dextrinoid basidiospores. The differences between the new species and phylogenetically related and morphologically similar species are discussed.
\end{abstract}

Key words - Phylogeny - polyporales - taxonomy - wood-decaying fungi

\section{Introduction}

The polypore genus Perenniporia Murrill (Polyporales, Basidiomycetes) is distributed in almost all the forests of the earth and very rich in species diversity. It has been extensively studied during last recent 20 years, because it includes some forest pathogens and medicinal fungi (Dai et al. 2007, 2009), and it is characterized by distinctly truncate, thick-walled cyanophilous and variably dextrinoid basidiospores and a dimitic structure with cyanophilous and variably dextrinoid skeletal hyphae. Until now, nearly 100 species are included in the genus (Gilbertson \& Ryvarden 1987, Hattori \& Lee 1999, Decock et al. 2000, 2001, 2011, Dai et al. 2002, Choeyklin et al. 2009, Cui \& Zhao 2012, Zhao et al. 2013, Decock \& Ryvarden 2013, 2015, Ryvarden \& Melo 2014, Viacheslav \& Ryvarden 2016).

During investigations on wood-inhabiting fungi in East Asia, two specimens were collected from northern Thailand and they match the characteristics of Perenniporia. To confirm their taxonomic affinity and the evolutionary relationships among representative species of Perenniporia, phylogenetic analysis was carried out based on ITS sequences. Both morphological and molecular data support these two samples represent a new species and it is described and illustrated in this paper.

\section{Materials \& methods}

\section{Morphological studies}

The specimens studied are deposited in the herbarium of the Institute of Microbiology, Beijing Forestry University (BJFC) and the Center of Excellence in Fungal Research, Mae Fah Luang University (MFLU). The microscopic procedure follows Zhao \& Cui (2013). Microscopic features, measurements and drawings were made from slide preparations stained with Cotton Blue and Melzer's reagent. In presenting the variation in the size of the spores, $5 \%$ of measurements were excluded from each end of the range, and were given in parentheses. In the text the following abbreviations were used: $\mathrm{KOH}=5 \%$ potassium 
hydroxide, $\mathrm{IKI}=$ Melzer's reagent, IKI $-=$ neither amyloid nor dextrinoid, $\mathrm{CB}=$ Cotton Blue, $\mathrm{CB}+=$ cyanophilous, $\mathrm{L}=$ mean spore length (arithmetic average of all spores), $\mathrm{W}=$ mean spore width (arithmetic average of all spores), $\mathrm{Q}=$ variation in the ratios of $\mathrm{L} / \mathrm{W}$ between specimens studied, $\mathrm{n}=$ number of spores measured from given number of specimens. Special color terms follow Petersen (1996).

\section{Molecular procedures and phylogenetic analyses}

The extraction of total genomic DNA from dried specimens and polymerase chain reactions follow Chen et al. $(2015,2016)$. Sequences newly generated in this study were aligned with additional related sequences downloaded from GenBank using Clustal X (Thompson et al. 1997) and manually adjusted in BioEdit (Hall 1999). Sequences of Donkioporia expansa (Desm.) Kotl. \& Pouzar and Pyrofomes demidoffii (Lév.) Kotl. \& Pouzar were used as outgroups following Zhao et al. (2013) in the phylogenetic analyses.

Maximum parsimony (MP) and Bayesian inference (BI) analyses were conducted for the datasets of ITS sequences. The best-fit evolutionary model was selected by hierarchical likelihood ratio tests (hLRT) and Akaike information criterion (AIC) in MrModeltest 2.2 (Nylander 2004) after scoring 24 models of evolution by PAUP* version 4.0b10 (Swofford 2002).

The MP topology and bootstrap values (MP-BS) obtained from 1000 replicates were performed using PAUP* version 4.0b10 (Swofford 2002). All characters were equally weighted and gaps were treated as missing. Trees were inferred using the heuristic search option with TBR branch swapping and 1000 random sequence additions. One thousand max-trees were set, branches of zero length were collapsed and all parsimonious trees were saved. Descriptive tree statistics tree length (TL), consistency index (CI), retention index (RI), rescaled consistency index (RC), and homoplasy index (HI) were calculated for each Maximum Parsimonious Tree (MPT) generated.

Bayesian phylogenetic inference and Bayesian posterior probabilities (BPP) were performed with MrBayes 3.1.2 (Ronquist \& Huelsenbeck 2003). Four Markov chains were run for five million generations until the split deviation frequency value $<0.01$, and sampled every 100 generation resulting in 90,000 trees. The first $25 \%$ of the sampled trees were discarded as burn-in and the remaining ones were used to reconstruct a majority rule consensus and calculate Bayesian posterior probabilities (BPP) of the clades.

Branches that received bootstrap support for MP and BI greater than or equal to 50\% (MPBS) and 0.95 (BPP) were considered as significantly supported, respectively.

\section{Results}

\section{Phylogeny}

Three newly generated ITS sequences are deposited at GenBank (www.ncbi.nlm.nih.gov/genbank/). The accession numbers of the used sequences in this study are labeled in the phylogenetic tree (Fig. 1). The ITS dataset has 24 taxa and resulted in an alignment of 608 character, of which 345 characters are constant, 38 are variable and parsimony- informative, and 225 are parsimony-informative. Maximum parsimony analysis yielded 6 equally parsimonious trees $(\mathrm{TL}=695, \mathrm{CI}=0.542, \mathrm{HI}=0.458, \mathrm{RI}=0.775, \mathrm{RC}=0.420)$. Best model for the ITS estimated and applied in the Bayesian analysis: $\mathrm{GTR}+\mathrm{I}+\mathrm{G}$, lset nst $=6$, rates $=$ invgamma; prset statefreqpr $=$ dirichlet $(1,1,1,1)$. BI resulted in the nearly congruent topology with MP analysis, with an average standard deviation of split frequencies $=0.007280$.

The phylogeny shows that the three newly sequenced specimens form a distinct lineage with full statistical support (100/1.00), and this lineage occupies a separate position from known species of Perenniporia (Fig. 1).

\section{Taxonomy}

Perenniporia chiangraiensis $\mathrm{F}$. Wu \& X.H. Ji, sp. nov.

Figs 2, 3

MycoBank number MB 819946; Facesoffungi number FoF 02917.

Holotype - Thailand. Chiang Rai, Doi Mae Salong, on angiosperm tree root, 22 July 2016, Dai 16637 (holotype in BJFC 022747, isotype in MFLU).

Etymology - Chiangraiensis (Lat.): referring to the locality, Chiang Rai of Thailand.

Fruiting body - Basidiocarps perennial, pileate, soft corky and without odour or taste when fresh, corky when dry. Pilei dimidiate to more or less fan-shaped, projecting up to $4 \mathrm{~cm}, 6 \mathrm{~cm}$ wide and $2 \mathrm{~cm}$ thick at base. Pileal surface clay-buff with distinctly concentric sulcate when fresh, becoming greyish brown when dry; margin acute. Pore surface white when fresh, colour unchanged when dry; sterile margin very narrow to almost lacking; pores circular, 6-8 per mm; dissepiments thick, entire. Context fawn, corky, up to $2 \mathrm{~mm}$ 
thick. Tubes distinctly stratified, corky, up to $18 \mathrm{~mm}$ long; old tubes cinnamon, new tubes white. Hyphal Structure - Hyphal system dimitic; generative hyphae bearing clamp connections; skeletal hyphae strongly dextrinoid, $\mathrm{CB}+$; tissues unchanged in $\mathrm{KOH}$. Context - Generative hyphae very infrequent, hyaline, thinwalled, occasionally branched, $1.5-2.5 \mu \mathrm{m}$ in diam; skeletal hyphae dominant, thick-walled with a narrow lumen to subsolid, occasionally branched, strongly flexuous, interwoven, $1.5-2.5 \mu \mathrm{m}$ in diam. TubesGenerative hyphae infrequent, hyaline, thin-walled, occasionally branched, $1-2 \mu \mathrm{m}$ in diam; skeletal hyphae dominant, thick-walled with a narrow lumen, frequently branched, strongly flexuous, interwoven, $1.5-2 \mu \mathrm{m}$ in diam. Dendrohyphidia present at dissepiments, hyaline, thin-walled; cystidia absent; cystidioles present, mostly fusiform, thin-walled, smooth, 12-16 × 4-6 $\mu \mathrm{m}$. Basidia barrel-shaped to pear-shaped, with four sterigmata and a basal clamp connection, 10-15 $\times 4-7 \mu \mathrm{m}$; basidioles in shape similar to basidia, but smaller. Basidiospores ellipsoid, truncate, hyaline, slightly thick-walled, smooth, IKI-, CB+, (3.2-)3.4-4(4.1) $\times(2-) 2.2-2.8(-2.9) \mu \mathrm{m}, \mathrm{L}=3.71 \mu \mathrm{m}, \mathrm{W}=2.48 \mu \mathrm{m}, \mathrm{Q}=1.44-1.54(\mathrm{n}=60 / 2)$.

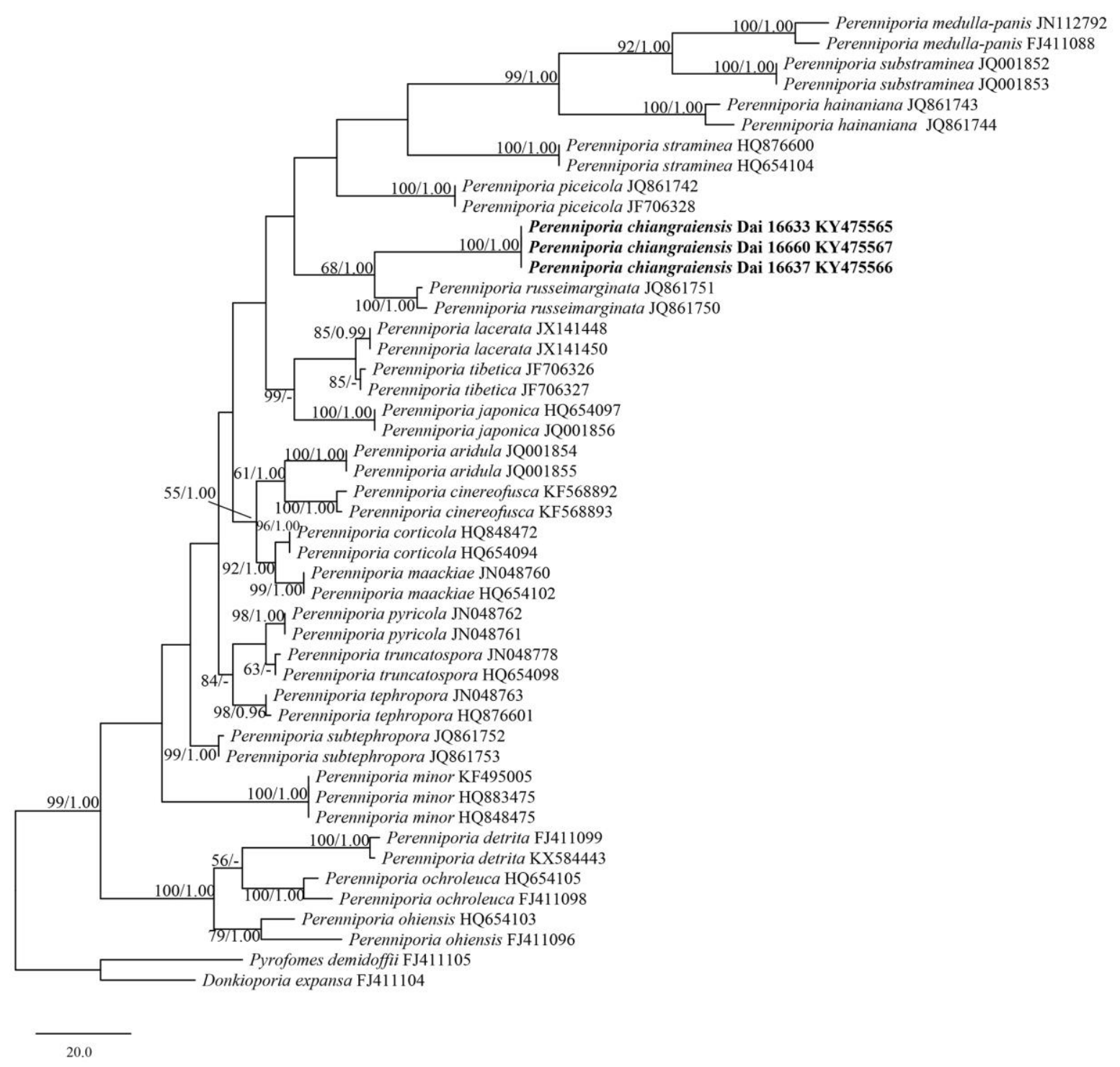

Fig.1 - Maximum parsimony strict consensus tree illustrating the phylogeny of Perenniporia based on ITS sequences. Branches are labeled with parsimony bootstrap proportions (before slanting line) higher than $50 \%$ and Bayesian posterior probabilities (after slanting line) more than 0.95 .

Notes - Perenniporia chiangraiensis is characterized by perennial, pileate basidiocarps, concentrically sulcate pileal surface, white pores, distinctly stratified tubes, a dimitic hyphal system with 
strongly dextrinoid skeletal hyphae, the presence of dendrohyphidia, and ellipsoid, truncate, thick-walled, non-dextrinoid, cyanophilous basidiospores.

Additional specimen examined - Thailand. Chiang Rai, Doi Mae Salong, on angiosperm tree root, 22 July 2016, Dai 16633 (BJFC 022743, MFLU) \& Dai 16660 (BJFC 022768, MFLU paratypes).

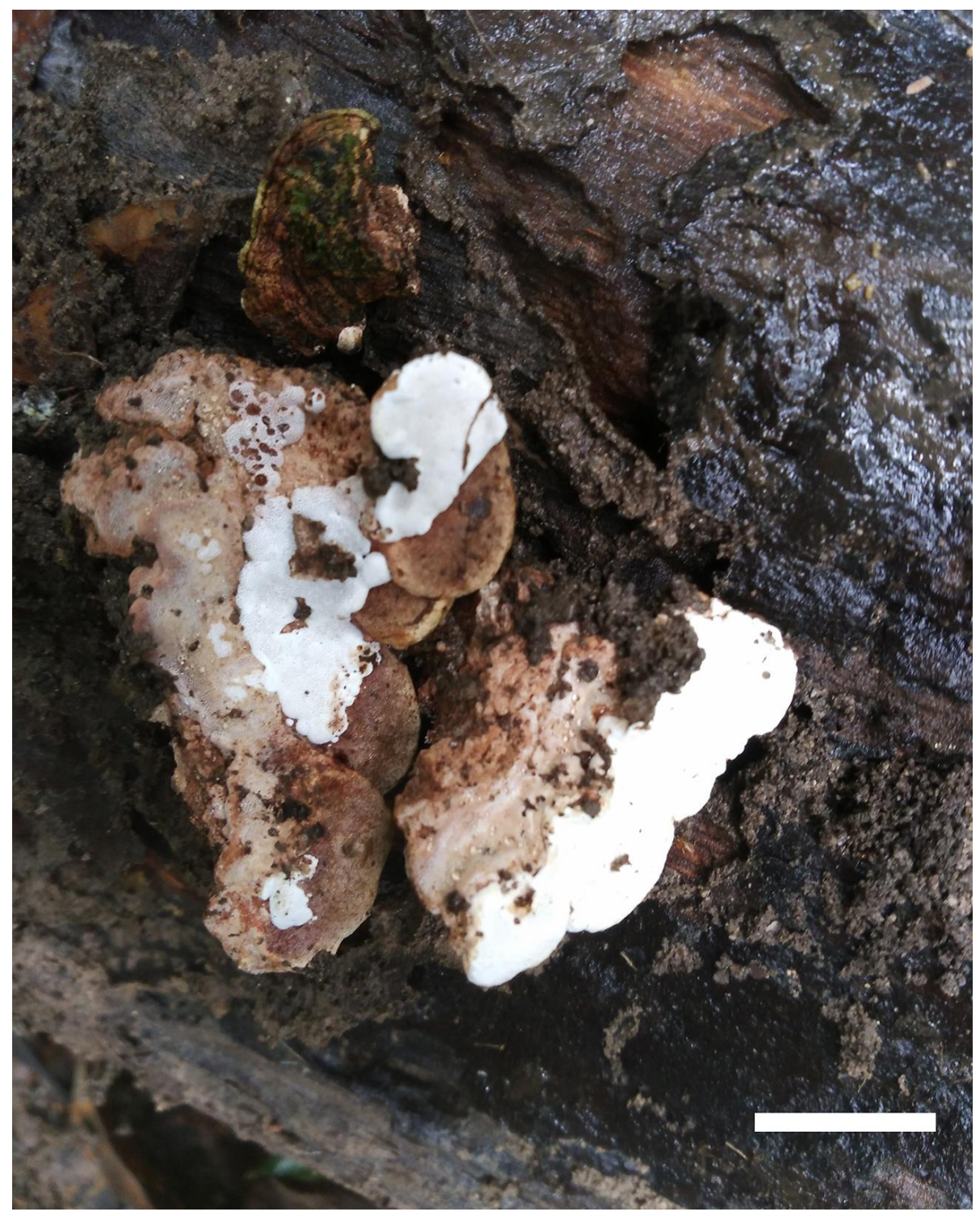

Fig.2 - Basidiomata of Perenniporia chiangraiensis (Holotype). - Scale bar $=1 \mathrm{~cm}$

\section{Discussion}

In the present study, a new polypore, Perenniporia chiangraiensis, is described based on phylogenetic analysis and morphological characters. The species has unique morphological characters in Polyporaceae and forms a distinct lineage within the Perenniporia clade.

Phylogenetically Perenniporia chiangraiensis is related to P. russeimarginata B.K. Cui \& C.L. Zhao (Fig. 1), but the latter has resupinate fruiting body, dextrinoid and bigger basidiospores $(4-5 \times 3-4 \mu \mathrm{m}$, Zhao \& Cui 2013).

Perenniporia chiangraiensis has soft fresh fruiting body, regular pores with thick and entire dissepiments, so these characters remind of Wrightoporia Pouzar, but basidiospores in Wrightoporia are ornamented and amyloid (Chen et al. 2016).

Perenniporia contraria (Berk. \& M.A. Curtis) Ryvarden resembles $P$. chiangraiensis in having pileate fruiting body with round pores as 6-8 per $\mathrm{mm}$, a dimitic hyphal structure with strongly dextrinoid skeletal hyphae, and cyanophilous, non-dextrinoid basidiospores (Decock et al. 2001), but the former species lacks dendrohyphidia and has thick-walled, non-truncate and bigger basidiospores (3.7-4.5 $\times 3-3.8 \mu \mathrm{m}$, Decock et al. 2001). 
Previously dendrohyphidia were reported in Perenniporia substraminea B.K. Cui \& C.L. Zhao, but it differs from $P$. chiangraiensis in having reupinate fruiting body, smaller pores (9-12 per $\mathrm{mm}$ ) and strongly dextrinoid basidiospores (Zhao et al. 2013).
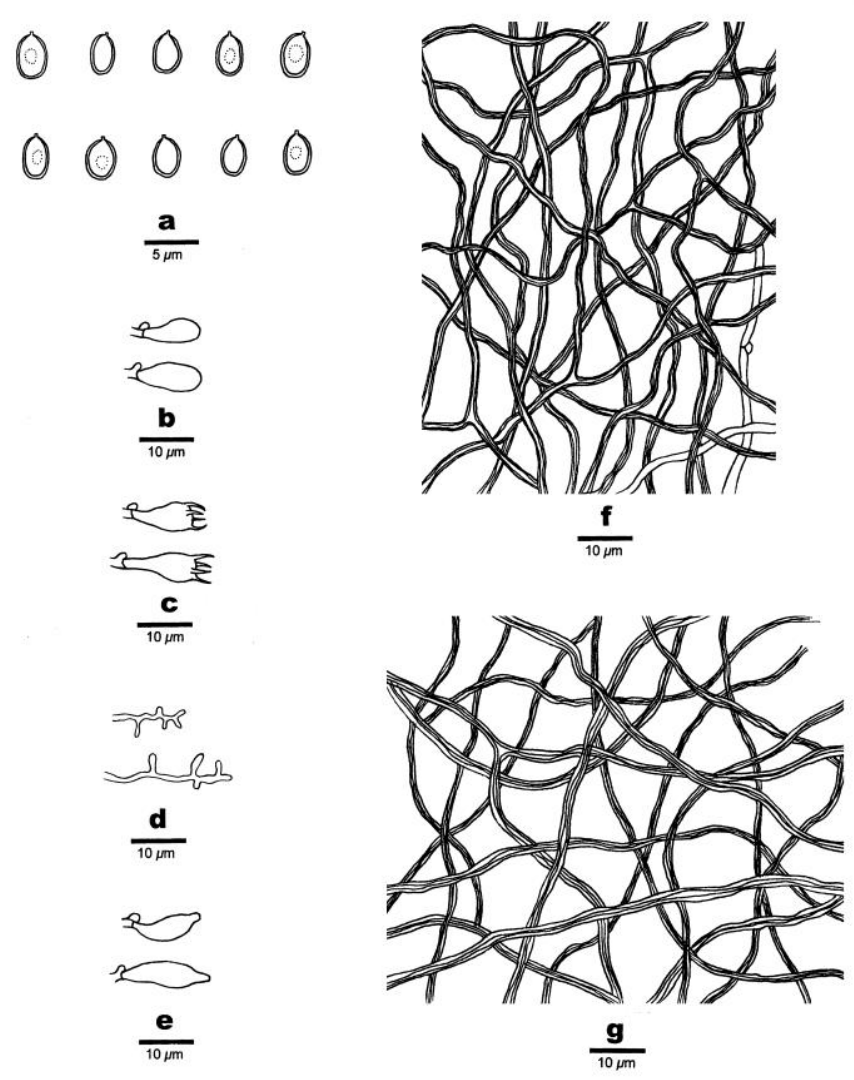

Fig.3 - Microscopic structures of Perenniporia chiangraiensis (Holotype). a. Basidiospores; b. Basidioles; c. Basidia; d. Cystidioles; e. Dendrohyphidia from dissepiments; f. Hyphae from tube trama; g. Hyphae from context.

\section{Acknowledgments}

We express our gratitude to Prof. Yu-Cheng Dai (BJFC, China) allowed us to study his specimens. The research is supported by the National Natural Science Foundation of China (Project Nos. 31530002).

\section{References}

Chen JJ, Cui BK, Dai YC. 2016 - Global diversity and molecular systematics of Wrightoporia s.l. (Russulales, Basidiomycota). Persoonia 37, 21-36.

Chen JJ, Cui BK, Zhou LW, Korhonen K, Dai YC. 2015 - Phylogeny, divergence time estimation, and biogeography of the genus Heterobasidion (Basidiomycota, Russulales). Fungal Diversity 71, 185-200.

Choeyklin R, Hattori T, Jaritkhuan S, Jones EBG. 2009 - Bambusicolous polypores collected in central Thailand. Fungal Diversity 36, 121-128.

Cui BK, Zhao CL. 2012 - Morphological and molecular evidence for a new species of Perenniporia (Basidiomycota) from Tibet, southwestern China. Mycoscience 53, 365-372.

Dai YC, Cui BK, Yuan HS, Li BD. 2007 - Pathogenic wood-decaying fungi in China. Forest Pathology 37, 105120.

Dai YC, Niemelä T, Kinnunen J. 2002 - The polypore genera Abundisporus and Perenniporia (Basidiomycota) in China, with notes on Haploporus. Annales Botanicci Fennici 39, 169-182.

Dai YC, Yang ZL, Cui BK, Yu CJ, Zhou LW. 2009 - Species diversity and utilization of medicinal mushrooms and fungi in China (Review). Int J Med Mushrooms 11, 287-302.

Decock C, Buchanan PK, Ryvarden L. 2000 - Revision of some Australasian taxa of Perenniporia (Basidiomycota, Aphyllophorales). Australian Syst. Bot. 13, 823-844.

Decock C, Figueroa H, Ryvarden L. 2001 - Studies in Perenniporia. Perenniporia contraria and its presumed taxonomic synonym Fomessubannosus. Mycologia 93, 196-204. 
Decock C, Mossebo DC, Yombiyeni P. 2011 - Studies in Perenniporia s. lat. (Basidiomycota). African taxa V: Perenniporia alboferruginea sp. nov. from Cameroon. Plant Ecol Evol 144, 226-232.

Decock C, Ryvarden L. 2015 - Studies in Perenniporia s.l. African taxa 9: Perenniporia vanhullii sp. nov. from open woodlands. Synopsis Fungorum 33, 43-49.

Decock C, Ryvarden L. 2013 - Neotropical Perenniporia: Perenniporia subovoidea sp. nov., new records of little known species, and a key to the species with resupinate basidiome. Cryptogamie Mycologie 146, 234-239.

Gilbertson RL, Ryvarden L. 1987 - North American polypores 2. Megasporoporia-Wrightoporia. Fungiflora, Oslo.

Hall TA. 1999 - Bioedit: a user-friendly biological sequence alignment editor and analysis program for windows 95/98/NT. Nucleic Acids Symp Ser 41, 95-98.

Hattori T, Lee SS. 1999 - Two new species of Perenniporia described from a lowland rainforest of Malaysia. Mycologia 91, 525-531.

Nylander JAA. 2004 - MrModeltest v2. Program distributed by the author. Evolutionary Biology Centre, Uppsala University.

Petersen JH. 1996 - Farvekort. The Danish Mycological Society's colour-chart. Foreningen til Svampekundskabens Fremme, Greve. pp. 1-6.

Ronquist F, Huelsenbeck JP. 2003 - MRBAYES 3: Bayesian phylogenetic inference under mixed models. Bioinformatics 19, 1572-1574.

Ryvarden L, Melo I. 2014 - Poroid fungi of Europe. Synopsis Fungorum 31, 1-455.

Swofford DL. 2002 - PAUP*: phylogenetic analysis using parsimony (*and other methods), version 4.0b10. Sinauer Associates, Sunderland.

Thompson JD, Gibson TJ, Plewniak F, Jeanmougin F, Higgins DG. 1997 - The Clustal_X windows interface: flexible strategies for multiple sequence alignment aided by quality analysis tools. Nucleic Acids Research $25,4876-4882$.

Viacheslav S, Ryvarden L. 2016 - Somebasidiomycetes (Aphyllophorales) from Mexico. Synopsis Fungorum $35,34-42$

Zhao CL, Cui BK. 2013 - Morphological and molecular identification of four new resupinate species of Perenniporia (Polyporales) from southern China. Mycologia 105, 945-958.

Zhao CL, Cui BK, Dai YC. 2013 - New species and phylogeny of Perenniporia based on morphological and molecular characters. Fungal Diversity 58, 47-60. 